\title{
25 Research Suare \\ Method to Determine the Statistical Technical Variability of SUV Parameters
}

Giulia Maria Rita De Luca ( $\nabla$ g.deluca@antoniusziekenhuis.nl)

Sint Antonius Ziekenhuis https://orcid.org/0000-0002-7485-2914

Jan Habraken

Sint Antonius Ziekenhuis

Original research

Keywords: Standard Uptake Value, PET quantification, variation in Standard Uptake Value

Posted Date: October 28th, 2021

DOI: https://doi.org/10.21203/rs.3.rs-929870/v1

License: (a) (i) This work is licensed under a Creative Commons Attribution 4.0 International License. Read Full License 


\section{Abstract}

Background: Some of the parameters used for the quantification of Positron Emission Tomography (PET) images are the Standardized Uptake Value (SUV)Max, SUVMean and SUVPeak. In order to assess the significance of an increasing or decreasing of these parameters for diagnostic purposes it is relevant to know their standard deviation. The sources of the standard deviation can be divided in biological and technical. In this study we present a method to determine the technical variation of the SUV in PET images.

Results: This method was tested on images of a NEMA quality phantom with spheres of various diameters with full-length acquisition time of $150 \mathrm{~s}$ per bed position and foreground to background activity ratio of $\mathrm{F}^{18}$-2-fluoro-2deoxy-D-glucose (FDG) of 10:1. Our method is based on dividing the full-length $150 \mathrm{~s}$ acquisition into subsets of shorter time length and reconstructing the images in the subsets. The SUVMax, Mean and Peak were calculated for each reconstructed image in a subset. The coefficient of deviation of the SUV parameters within each subset has then been used to estimate the expected standard deviation between images at $150 \mathrm{~s}$ reconstruction length. We report the largest technical variation of the SUV parameters for the smallest sphere, and the smallest variation for the largest sphere. The expected variation at 150 s reconstruction length does not exceed $6 \%$ for the smallest sphere and $2 \%$ for the largest sphere.

Conclusions: With the presented method we are able to determine the technical variation of SUV. The method enables us to evaluate the effect of parameter selection and lesion size on the technical variation, and therefore to evaluate its relevance on the total variation of the SUV value between studies.

\section{Introduction}

Positron Emission Tomography (PET) has become an indispensable diagnostic tool over the last decades. Computed Tomography (CT) is added to the PET modality for the purpose of attenuation correction and furthermore PET-CT imaging provides a combined view of functional and morphological information.

The radio-ligand $\mathrm{F}^{18}$-2-fluoro-2-deoxy-D-glucose (FDG) has ensured the success of PET-imaging. The glucose component of the molecule provides a higher uptake of FDG in malignant than in healthy cells [1], the $F^{18}$ component provides the detectability in the PET-CT system.

PET-CT images can be reported visually by the nuclear medicine physician, however an important advantage of PET imaging is that the uptake can be quantified in absolute measures. Quantification of FDG PET enables the staging of cancer and the comparison of follow-up studies to track the evolution of cancer and response to tumour therapy [2]. An important factor in quantification is to minimize the inter- and intra-observer variability as much as possible.

One of the measures for the comparison of $\mathrm{F}^{18}$-FDG PET images is the Standardized Uptake Value (SUV). The most used SUV parameters are the SUVMax and the SUVMean. In the SUVMax only the voxel with the highest uptake is considered, while in the SUVMean all the voxels in a certain region or volume are taken into account. The SUVMax has a low inter- and intra-observer variability but a high technical statistical variation. The SUVMean on the other hand has a lower technical variation but a higher inter- and intra-observer variability, since the thresholds for the borders of the volume are a determining factor of the result. The SUVPeak is introduced as a "best of both worlds" parameter because it includes the voxels in a fixed limited volume around the voxel with the maximum value and it 
might improve reproducibility for SUV measurements especially of the most metabolically active tumour regions [3].

The proposed framework for PET Response Criteria in Solid Tumours (PERCIST) suggests to consider a 30\% change in SUV as significant variation of tumour activity [4]. Decreasing the variability of the SUV quantification will enable the detection of smaller significant changes and therefore enable an earlier detection of degeneration or an effect of therapy. Limiting the variations and standardizing the process as much as possible is therefore essential.

In test-retest studies, patients are injected and scanned twice to assess the variation of the complete PET imaging process. However, to be able to further reduce the variation efficiently it is important to know from which components the total variation is built up.

Theoretically, the variation in a PET measurement consists of biological variability and technical variability [5]. Biological variability arises from variations in blood glucose, paravenous administration of FDG and FDG uptake and can be minimized by standardizing the patient preparation with a protocol defining diet and uptake time. For what concerns the technical variability, studies have shown that important factors effecting the technical variation of the SUV are variation in image reconstruction and scanner characteristics $[6,7,8]$. Standardizing the positioning of the patient, acquisition, reconstruction and quantification protocol and PET-system calibration will reduce the technical variation. However a certain amount of technical variation is unavoidable due to the statistical variations of the $\mathrm{F}^{18}$ decay and the image reconstruction.

We define this as the "statistical technical variation", which is the part of the technical variation that cannot be minimized by standardization but is intrinsically present on statistic grounds. Knowing the statistical technical variation is important because it gives us insight in the origin of the total variation.

In order to be able to evaluate the impact of an increase of the dose on the total variation it is necessary to be able to calculate its effect on the technical variation. Since the statistical technical variation differs for the several SUV parameters it is important to be able to specifically quantify it. Furthermore the statistical technical variability can be scanner- and reconstruction-specific and will be dependent on the size of the lesions. The assessment of the statistical technical variation gives insight in its contribution to the total variation and enables the effect of several parameters on the technical variation.

In this study we present a method to estimate the statistical technical variation on different SUV parameters and lesion sizes of images acquired with the same scanner and reconstructed with the same method. The basics of the method is that one PET acquisition is divided into a number of time-frames and that the variation between the SUV quantification of the separate time-frames is used to estimate the standard deviation of the total acquisition. The proposed method determines the standard deviation of SUV parameters such that the relevance of technical statistical SUV changes in clinical practice can be established in the context of other fluctuations.

The method is described, validated and applied on a $150 \mathrm{~s}$ acquisition of the image quality phantom with a foreground to background activity ratio of 10:1 as example of application. The values and standard deviations of the SUVMax, SUVMean and SUVpeak of the several spheres in the phantom are presented and discussed.

\section{Material And Methods}


The method that we describe in this paper for estimating the statistical technical variation of SUV parameters between PET images includes three main steps:

- acquisition of the dataset

- generation and reconstruction of subset with shorter acquisition frames

- calculation of the variation in the subsets, and traslation to the variation of the original dataset

To test the validity of our method, the variation is calculated based on subsets with different acquisition lengths.

\section{Image acquisition and reconstruction}

A NEMA NU2-2007 image quality phantom was imaged on a Philips Gemini TF PET/CT system (Philips Healthcare, Andover, MA). PET reconstructions were made using scanner's default Ordered Subset Expectation Maximization (OSEM) reconstruction algorithm with 33 subsets, 3 iterations, matrix size of $144 \times 144$, and voxels of $4 \times 4 \times 4 \mathrm{~mm}$. No Gaussian filter was applied. The reconstruction was corrected for geometrical response and detector efficiency (normalization), random coincidences, scatter and attenuation. Data were stored in list-mode, to be able to reconstruct subsets with different acquisition times. All list-mode reconstructions were decay-corrected to the start time of the acquisition.

The phantom acquisitions were made according to the requirements for the EANM/EARL FDG-PET/CT accreditation [9]. The phantom was composed by a fillable torso compartment acting as background, by a cylindrical insert in the centre of the torso compartment and by 6 fillable spheres of different diameters (10 mm, 13 $\mathrm{mm}, 17 \mathrm{~mm}, 22 \mathrm{~mm}, 28 \mathrm{~mm}$ and $37 \mathrm{~mm}$ ) placed around the central insert. The fillable torso compartment and the spheres have been filled with a solution of water and $\mathrm{F}^{18}$-FDG. At the starting moment of the scan the activity concentration was $2.10 \mathrm{MBq} / \mathrm{ml}$ in the torso background compartment and $20.04 \mathrm{MBq} / \mathrm{ml}$ in the spheres, resulting in an actual sphere to background ratio of 9.6:1 (aim is 10:1). [10]

The original dataset was acquired with $150 \mathrm{~s}$ frame duration. The total acquisition time was 10 minutes (600 s, 4 bed positions). The full-length $150 \mathrm{~s}$ list-mode acquisition was divided into subsets of shorter time length varying from $4 \mathrm{~s}$ to $30 \mathrm{~s}$. An attenuation corrected reconstruction was performed for different reconstruction lengths, generating as many images as possible per subset, without using the same coincidences by varying the starting time of the reconstruction. For example, for the first subset (4 s reconstruction length), the first image was reconstructed using the coincidences recorded between 0 and 4 seconds, the second image by using the coincidences recorded between 5 and 8 seconds and so on, varying the starting moment of the reconstruction, generating a total of 37 images. The longest frame length was $30 \mathrm{~s}$, generating a subset of 5 images. A total of 14 subsets was generated, of respectively $4 s, 6 s, 8 s, 10 s, 12 s, 15 s, 17 s, 19 s, 20 s, 22 s, 24 s$ 26s, 28s and 30 s acquisition length.

The Philips reconstruction software automatically corrected each reconstruction for the decay of ${ }^{18} \mathrm{~F}$ (half-life of 109.7 minutes [11]), compensating the time difference between the start of the study and the start of the reconstruction by using an opportune scaling factor.

\section{Image analysis}

The datasets were analysed using a Python 3.7.0 script (default, Jun 28 2018, 08:04:48) [MSC v.1912 64 bit (AMD64)]. The algorithm automatically detected the spheres and their central 2D plane. Different SUV parameters 
were calculated in each image of the subsets:

- SUVMax 2D: the maximum voxel value in the central 2D plane;

- SUVMax 3D: maximum voxel value in each sphere;

- SUVMean 2D: the average value of the voxels in the central 2D plane of each sphere;

- SUVMean 3D: the average value of the voxels in each sphere.

The SUVMean 2D and 3D were calculated considering the complete 2D central plane or 3D volume, respectively, without using thresholding techniques based on pixel values or on a percentage of the maximum value.

- SUVPeak: the average value within a $1 \mathrm{~cm}^{3}$ sphere centred in the maximum value of the sphere [12]. The algorithm fitted the sphere, found the maximum voxel value in the 3D volume, used this voxel as the centre of a spherical Region of Interest (ROI) of $1 \mathrm{~cm}^{3}$ and calculated the average value within the $1 \mathrm{ml}$ sphere.

The SUV values were calculated per image in a subset. The SUV values populations have been tested for normality with a Kolmogorov-Smirnov test and all subsets matched the characteristics of a normal distribution. The SUV parameters of the different images in a subset were averaged and their standard deviation was calculated. The coefficient of variation of the SUV parameters was calculated as the standard deviation divided by their average value multiplied by 100 .

In our measurements we can assume a random sampling model, with no correlations, for independent and identically distributed random measurements. The different subsets do not differ in activity nor voxel dimensions and the quantification of the SUV parameters has been done by using the same ROI dimension. We can therefore describe the ratio of the standard deviations SD of two independent repetitions of PET measurements as:

$$
\frac{S D 1}{S D 2}=\left(\frac{R L 1}{R L 2}\right)^{-\frac{1}{2}}
$$

With SD1 and SD2 being the standard deviations and RL1 and RL2 being the reconstruction lengths. [7]. By setting the measured coefficient of variation of the SUV in a subset as SD1 and the length of the reconstruction of the specific subset as RL1, it is possible to estimate the variation SD2 between repetitions of scans at the total acquisition time RL2=150 s according to:

$$
S D 2=S D 1\left(\frac{R L 1}{R L 2}\right)^{\frac{1}{2}}
$$

Formula 2 was used to calculate the estimated SD of the SUV at reconstruction length $S D 2=150$ s using each subset of the dataset. Since we divided our acquisition into 14 different subsets, we could calculate 14 different estimations of the SD2. We validated our method by testing whether the value of the estimated SD2 was independent of the acquisition length of the images in the subset.

Furthermore, the results were tested to verify if there is a significant difference in SUV between adjacent spheres. The population of two adjacent spheres $(10 \mathrm{~mm}$ and $13 \mathrm{~mm}, 13 \mathrm{~mm}$ and $17 \mathrm{~mm}, 17 \mathrm{~mm}$ and $22 \mathrm{~mm}, 22 \mathrm{~mm}$ and $28 \mathrm{~mm}, 28 \mathrm{~mm}$ and $37 \mathrm{~mm}$ ) were tested by using a two-sample t-test assuming unequal variances with a significance level alpha=0.05. 


\section{Results}

After generating and reconstructing the images in the subsets with shorter acquisition frames, the SUVMax 2D and 3D, the SUVMean 2D and 3D and the SUVPeak were calculated for the 6 spheres in each image of a subset. The SUV parameters from all the images per sphere were averaged and their standard deviation was estimated. The average value was plotted as a function of the sphere diameter, obtaining a recovery curve based on SUVMax 2D and 3D, the SUVMean 2D and 3D and the SUVPeak. The results are shown in Fig. 1 as examples for the $4 \mathrm{~s}$ (blue line), $15 \mathrm{~s}$ (red line) and $30 \mathrm{~s}$ (yellow line) subsets.

For the larger spheres, for the SUVMax and the SUVPeak parameters, the shorter acquisition lengths tend to have a higher average value, as shown for the three representative datasets in Fig. 1.

Fig. 2 to 6 show the coefficient of variation of the measured SUV parameters. The coefficient of variation of the SUV is plotted as a function of the reconstruction length for the different spheres. We show the results for the spheres with $10 \mathrm{~mm}$ and $37 \mathrm{~mm}$ diameter as representative results.

After calculating the variation in the subsets, the results were used to traslate the results to the coefficient of variation of the original dataset. Using Formula 2 we derived the expected coefficient of variation for the reconstruction length of $150 \mathrm{~s}$ by using the coefficients of variation of the subsets with $4 \mathrm{~s}$ to $30 \mathrm{~s}$ reconstruction length. The estimated variation of the SUVMax 2D and 3D, SUVMean 2D and 3D and SUVPeak at $150 \mathrm{~s}$ reconstruction length are plotted in Fig. 7 for the $10 \mathrm{~mm}$ diameter sphere and in Fig. 8 for the 37 mm diameter sphere as examples. The dots show the calculated coefficient of variation of the SUV at 150 s reconstruction length (SD2 in Formula 2) using each different subset for the estimation (SD1 in Formula 2). Each figure also reports the average value (red line) plus and minus the standard deviation (blue lines) of the results, showing that the acquisition length of the images in the subset has no structural effect on the estimated standard deviation at 150 seconds.

The estimated average coefficient of variation of the SUVMax2D and 3D, SUVMean2D and 3D and SUVPeak at 150 $s$ reconstruction length and its standard deviation has been estimated for spheres of different diameters as plotted in Fig. 7 and 8 for the $10 \mathrm{~mm}$ and $37 \mathrm{~mm}$ spheres (red and blue lines respectively). The average estimated coefficients of variation are summarized in Table 1. The spheres reporting a significant difference between them ( $p$ value t-test<0.05) are underlined and in bold in Tab.1.

Tab.1: Average estimated SD for SUV Max, Mean and Peak for different spheres. 


\begin{tabular}{|c|c|c|c|c|c|c|}
\hline & $\mathrm{d}_{\text {sphere }}=10 \mathrm{~mm}$ & $d_{\text {sphere }}=13 \mathrm{~mm}$ & $\mathrm{~d}_{\text {sphere }}=17 \mathrm{~mm}$ & $d_{\text {sphere }}=22 \mathrm{~mm}$ & $\mathrm{~d}_{\text {sphere }}=28 \mathrm{~mm}$ & $\begin{array}{l}d_{\text {sphere }}=37 \\
\mathrm{~mm}\end{array}$ \\
\hline $\begin{array}{l}\text { Est. SD } \\
\text { SUVMax } \\
\text { 2D }\end{array}$ & $5.6 \pm 1.1 \%$ & $3.8 \pm 1.2 \%$ & $3.4 \pm 0.8 \%$ & $3.3 \pm 0.7 \%$ & $2.9 \pm 0.9 \%$ & $1.6 \pm 0.3 \%$ \\
\hline $\begin{array}{l}\text { Est. SD } \\
\text { SUVMax } \\
\text { 3D }\end{array}$ & $5.5 \pm 1.1 \%$ & $3.8 \pm 1.2 \%$ & $3.4 \pm 0.8 \%$ & $3.1 \pm 0.7 \%$ & $2.6 \pm 0.6 \%$ & $1.9 \pm 0.6 \%$ \\
\hline $\begin{array}{l}\text { Est. SD } \\
\text { SUVMean } \\
\text { 2D }\end{array}$ & $5.0 \pm 1.0 \%$ & $3.7 \pm 1.1 \%$ & $2.8 \pm 0.5 \%$ & $2.2 \pm 0.4 \%$ & $1.7 \pm 0.4 \%$ & $0.9 \pm 0.3 \%$ \\
\hline $\begin{array}{l}\text { Est. SD } \\
\text { SUVMean } \\
\text { 3D }\end{array}$ & $5.0 \pm 0.9 \%$ & $3.6 \pm 0.9 \%$ & $2.5 \pm 0.6 \%$ & $1.6 \pm 0.3 \%$ & $1.3 \pm 0.3 \%$ & $0.8 \pm 0.2 \%$ \\
\hline $\begin{array}{l}\text { Est. SD } \\
\text { SUVPeak }\end{array}$ & $5.5 \pm 1.1 \%$ & $3.6 \pm 0.8 \%$ & $2.8 \pm 0.6 \%$ & $2.9 \pm 0.7 \%$ & $2.8 \pm 0.8 \%$ & $1.7 \pm 0.6 \%$ \\
\hline
\end{tabular}

The data were statistically analysed to verify if the difference between the estimated SD of the SUV parameters was significant between spheres (same parameter, different sphere diameter, so difference between columns in Tab.1) and between SUV parameters (different SUV parameter, same sphere diameter, so difference between rows in Tab.1).

Concerning the differences between spheres, we report that the difference between the estimated coefficient of variation of the SUV parameters of the sphere with $d=10 \mathrm{~mm}$ and $d=13 \mathrm{~mm}$ and with $\mathrm{d}=28 \mathrm{~mm}$ and $\mathrm{d}=37 \mathrm{~mm}$ is significant for SUVMax, Mean and Peak. For SUVPeak, the difference between the estimated coefficient of variation of the sphere with $d=13 \mathrm{~mm}$ and $d=17 \mathrm{~mm}$ is also significant. The difference between estimated coefficient of variation of the SUVMean $2 \mathrm{D}$ and $3 \mathrm{D}$ is significant between each sphere diameter.

Concerning the differences between SUV parameters, we report no significant difference between the estimated coefficient of variation of the SUV parameters of the two smaller spheres ( $d=10,13 \mathrm{~mm})$. The values of the SUVMean 3D are significantly lower than the values of the SUVMax 2D, 3D and SUVPeak for the four larger spheres $(d=17,22,28,37 \mathrm{~mm})$. The SUVPeak significantly differs from SUVMax 2D and 3D for $\mathrm{d}=17 \mathrm{~mm}$ and for SUVMean $2 \mathrm{D}$ and $3 \mathrm{D}$ for $\mathrm{d}=22,28,37 \mathrm{~mm}$.

\section{Discussion}

This study describes a methodology to estimate the statistical technical variability SUV parameters in PET images of different lesion sizes acquired with the same scanner and reconstructed with the same method. The proposed method determines the coefficient of variation of SUV parameters, which is a part of the total variation and provides an estimate of the relevance of the statistical technical variation in SUV values in the clinical practice. The value of this method is that it enables the possibility of estimate the influence of lesion size and choice of SUV parameter on the total variation.

The method divides the total acquisition into subsets at different timeframes and estimates the expected coefficient of variation of the total acquisition using the standard deviation within the subsets. The method was 
tested on a 150 s acquisition with a foreground to background activity ratio of 10:1.

The article shows how subsets of an original scan can be used to estimate the technical variation between images at different reconstruction lengths.

In Fig. 1 the calculated values of the SUV parameters are shown for all spheres and for all acquisition lengths of the timeframes in the subset. We report higher average SUV values for shorter acquisition lengths. This can be explained by the fact that at shorter acquisition lengths, when the images are noisier, the chance is bigger that a single voxel or group of voxels will have a higher value due to the a higher statistical variation. This effect occurs with the SUVMax and the SUVPeak, but not at the SUVMean where all the voxels in the regions are used for the calculation.

Figure 2 to 6 show the coefficient of variation of the SUV parameters as a function of the reconstruction length for the different spheres. This test can provide an expectation of the variation for noisier images (images with high standard deviation between voxels) or for small lesions. In our case we observe that the variation is higher for shorter reconstruction lengths, suggesting that the contribution of the technical variation might be higher, for example, for images acquired with a shorter acquisition time or with a low counts emitter. The higher variation at shorter reconstruction lengths reaches values up to $30 \%$, in our case, for the sphere with $10 \mathrm{~mm}$ diameter. This suggests that, when performing quantification of PET images on small lesion, the effect of the technical variability evaluated with this method might not be negligible when compared with the variation used for diagnostical purposes. Our method might also be used to define the minimal required acquisition length: when the technical statistical variation of the SUV has become negligible to the test-retest variation, a longer acquisition time might not add value. Fig. 7 and 8 show that the choice of the length of the subset to use for the estimation is not expected to create a bias in the estimation of the coefficient of variation of the full-length dataset.

In Table 1 we report the result of the estimated standard deviation of the SUV quantifications. We report significant differences in variation for different sphere dimensions. The difference is always significant for each SUV parameter for the smallest (diameter of $10 \mathrm{~mm}$ ) and the largest (diameter of $37 \mathrm{~mm}$ ) sphere. The difference is significant between all spheres for the SUVMean. The values are typically ranging from $5 \%$ for the $10 \mathrm{~mm}$ sphere to $1 \%$ for the $37 \mathrm{~mm}$ sphere. We notice that this is in accordance with the range reported for simulated data [13]. The maximum expected variation between images, for any estimated parameter, did not exceed the $6 \%$ for the smallest object (sphere of diameter $10 \mathrm{~mm}$ ) and the $2 \%$ for the largest object (sphere of diameter $37 \mathrm{~mm}$ ) for an acquisition length of $150 \mathrm{~s}$. This provides an indication of the contribution of the technical statistical variation when the same scanner is used, with equal reconstruction length and activity, and can be compared with the variation measured in FDG PET test-retest studies reporting a typical variation of approximately $10 \%[14,15,16]$. Our study is not a testretest study and aims to quantify the intrinsic statistical variation to expect when (ideally) repeating exactly the same acquisition without changing any external factors if not the statistical ones related to the characteristics of the emitters. The variation measured in our study is smaller than the one typically measured in a test-retest study due the fact that we do not have to deal with other factors, as for example repositioning of the patient or phantom and reinjection of the activity.

Nevertheless, it is important to notice that the value of the estimated technical statistical variation calculated for our scanner and reconstruction method is not directly translatable to other centres. The variability in the calculation of SUV parameters inhibits the direct comparison of these values. [17] Other factors introducing variation of the technical components are reconstruction protocols, analysis methods and scan duration, and their influence is too 
prominent for a direct comparison of the absolute values of the variation. [18] A simple method as the one described in this article can be routinely implemented to identify the contribution of the technical variation between PET images, in order to take into account of it during quantifications and comparisons of images for diagnostic purposes.

The SUV parameters (Max, Mean and Peak) presents some significant differences for the same sphere diameter. For what concerns the smaller spheres $(\mathrm{d}=10,13 \mathrm{~mm})$, the averaging step introduced in the calculation of SUVMean and Peak does not provide a significant difference in the coefficient of variation in our measurements for such small lesions. For larger lesions the difference between the variation in SUV Mean 3D and SUV Peak is significant. This suggests that the dimension of the ROI used for the averaging has a significant effect on the SUV quantification and that a too large ROI might flatten the results. It is worth reminding that our definition of SUVMean was based on the knowledge of the objects that were measured because the ROI was defined as a sphere of diameter equal to the nominal diameter of the imaged sphere. This is not always possible during the analysis of images for diagnosis purposes. In that case another definition of SUVMean must be used and the variation between measurements might be expected to increase $[12,14,15]$. Other factors present in clinical practice, as glucose blood levels, velocity of FDG uptake in the lesions or weight recording can increase the SUV variation in diagnostic images of patients. [19, 20, 21, 22, 23]

Another method to estimate the technical statistical variation would be to acquire a dataset with a longer acquisition time in comparison with the acquisition time used for diagnostic and generate subsets of the long acquisition with time length similar to the one used for diagnostic. This could be a more direct way to measure the variation, possibly less susceptible to low photon statistics as we report in images reconstructed with a short reconstruction length. A similar approach has been shown in [7] for SUVMax and Mean for reconstruction length of 5 minutes with variation between 11.2-1.2\% depending on the filter, type of acquisition (2D or 3D) and parameter (Max or Mean) used.

For this study we worked with a foreground to background activity ratio of 10:1. In order to further verify the method with other uptakes it would be possible to repeat the study with other ratio's, as for example 5:1 and 2.5:1. Furthermore, the acquisitions could be repeated after a certain amount of hours in order to analyse the variation with other levels of noise. As previously discussed, a higher coefficient of variation is to be expected for noisier images.

\section{Conclusion}

In this study we present a method to estimate the statistical technical variation of different SUV parameters. We used the method to estimate the statistical technical variation of SUV for different lesion sizes. The method shows that the coefficient of variation of SUVMax, SUVMean and SUVPeak varies as a function of the dimension of the objects in the imaged phantom and ranges between 5 and $6 \%$ for the smallest sphere (diameter of $10 \mathrm{~mm}$ ) and between 0.9 and $2 \%$ for the largest sphere (diameter of $37 \mathrm{~mm}$ ). The coefficient of variation reaches values up to $30 \%$ for shorter acquisition lengths (in the order of $4 \mathrm{~s}$ ), suggesting that the variation might become not negligible for noisier images with low counts. The difference between SUV parameters (Max, Mean and Peak) was not significant for the smaller spheres. The variation in SUVMean 3D was significantly lower for the larger spheres in comparison with the variation of the other parameters. Our method can be used routinely to provide insight into the statistical technical variation of a SUV quantification of images acquired with the same scanner and reconstruction parameter. 


\section{Declarations}

\section{Availability of data and materials}

The datasets used and/or analysed during the current study are available from the corresponding author.

\section{Acknowledgements}

The authors thank Ronald Boellaard, Amsterdam University Medical Centres, Location VUMC, for reading the manuscript and providing valuable feedback. The authors thank Olga Beregovaya, St. Antonius hospital, for her help with the measurements and the reconstructions.

\section{Funding}

Not applicable

\section{Contributions}

The authors read and approved the final manuscript.

\section{Ethics declarations}

Not applicable

\section{Consent for publication}

Not applicable

\section{Competing interests}

The authors declare that they have no competing interests.

\section{References}

1. Warburg O., "On the origin of cancer cells", Science 1956; 123:309-314

2. C.J. Hoekstra et al., "Monitoring response to therapy in cancer using [18F]-2-fluoro-2-deoxy-D-glucose and positron emission tomography: an overview of different analytical methods", European Journal of Nuclear Medicine June 2000 vol 27(6)

3. Lodge M.A. et al., "Noise Considerations for PET Quantification Using Maximum and Peak Standardized Uptake Value", Journal of Nuclear Medicine July 2012 vol 53 (7) 1041-1047

4. Walh R.L., "From RECIST to PERCIST: Evolving Considerations for PET Response Criteria in Solid Tumors", J Nucl Med May 2009 vol 50 (1) 122S-150S

5. Boellaard R., "Standards for PET image acquisition and quantitative data analysis. J Nucl Med. 2009 vol 50 (suppl 1) 11S-20S.

6. Higdon R. "Experimental Design, Variability", In: Dubitzky W., Wolkenhauer O., Cho KH., Yokota H. (eds) Encyclopedia of Systems Biology (2013) Springer, New York, NY.

7. Doot R.K. et al., "Instrumentation factors affecting variance and bias of quantifying tracer uptake with PET/CT", Med Phys. 2010 vol 37 6035-6046. 
8. Fahey F.H. et al., "Variability in PET quantitation within a multicenter consortium. Med Phys. 2010 vol 37 3660-3666.

9. R. Boellaard et al., “FDG PET/CT: EANM procedure guidelines for tumour imaging: version 2.0”, European Journal of Nuclear Medicine and Molecular Imaging 2015 vol 42 328-354

10. A. Kaalep at al., "EANM/EARL FDG-PET/CT accreditation - summary results from the first 200 accredited imaging systems", European Journal of Nuclear Medicine and Molecular Imaging 2018 Mar vol 45(3) 412-422

11. A.S. Keverling Buisman, “Handboek Radionucliden”, BetaText v.o.f., 2015

12. Joo Hyun O., “Practical PERCIST: A Simplified Guide to PET Response Criteria in Solid Tumors 1.0”, Radiology. August 2016 vol 280(2) 576-584

13. Boellaard R. "Effects of Noise, Image Resolution, and ROI Definition on the Accuracy of Standard Uptake Values: A Simulation Study", J Nucl Med 2004 vol 45 1519-1527

14. Abgral R. et al., "Comparison of prognostic value of tumor SUL-peak and SUV-max on pretreatment FDGPET/CT in patients with HNSCC", J Nucl Med May 2013 vol 54(2) 513

15. Julyan, P.J., "SUVpeak: a new parameter for quantification of uptake in FDG PET", Nucl Med Com April 2004 vol 25(4) 407

16. Lodge M.A., “Repeatability of SUV in Oncologic 18F-FDG PET”, J Nucl Med. Apr 2017 58(4) 523-532

17. Weber W.A. et al., "Reproducibility of metabolic measurements in malignant tumors using FDG PET" J Nucl Med 1999 vol 40 1771-1777.

18. Nahmias C. et al., "Reproducibility of standardized uptake value measurements determined by 18 F-FDG PET in malignant tumors. J Nucl Med. 2008 vol 49 1804-1808.

19. Pierce L.A. et al., "A Digital Reference Object to Analyze Calculation Accuracy of PET Standardized Uptake Value", Radiology 2015 vol 277 (2)

20. Syahir M. et al, "Impact of PET/CT system, reconstruction protocol, data analysis method, and repositioning on PET/CT precision: An experimental evaluation using an oncology and brain phantom", Med Phys. 201744 vol 12 6413-6424.

21. V. Kumar et al., "Variance of Standardized Uptake Values for FDG-PET/CT Greater in Clinical Practice than Under Ideal Study Settings" Clin Nucl Med. 2013 Mar; 38(3): 175-182.

22. Lodge M.A. et al., "A PET study of 18FDG uptake in soft tissue masses” Eur J Nucl Med. 1999;26:22-30

23. Fahey F.H. et al., "Variability in PET quantitation within a multicenter consortium” Med Phys. 2010;37:36603666

\section{Figures}



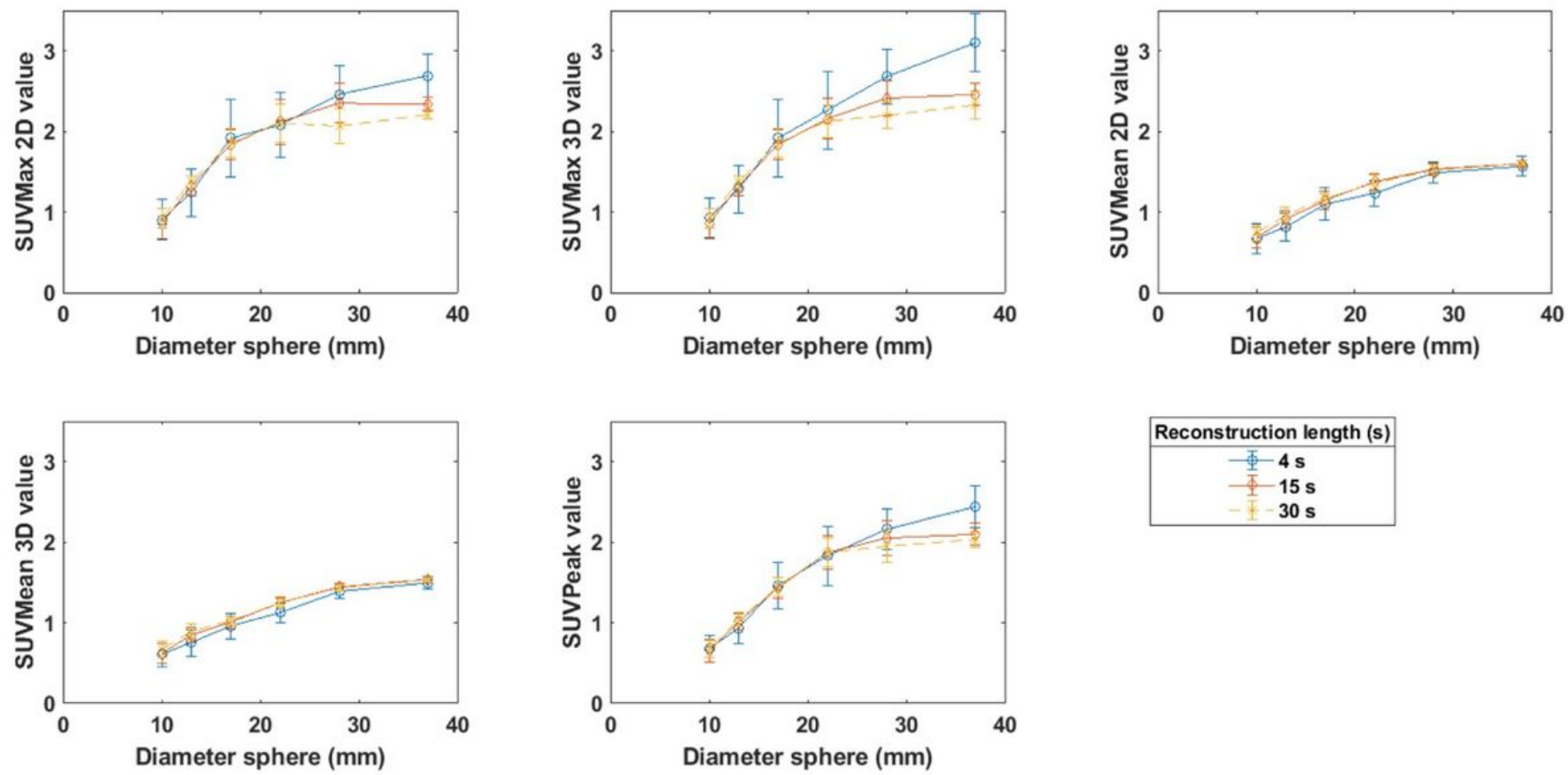

\section{Figure 1}

Average SUVMax 2D and 3D, SUVMean 2D and 3D and SUVPeak values $(\mathrm{kBq} / \mathrm{ml})$ indicates by circles and the corresponding standard deviations indicated by vertical bars for spheres with different volume for $4 \mathrm{~s}, 15 \mathrm{~s}$ and $30 \mathrm{~s}$ reconstruction lengths.
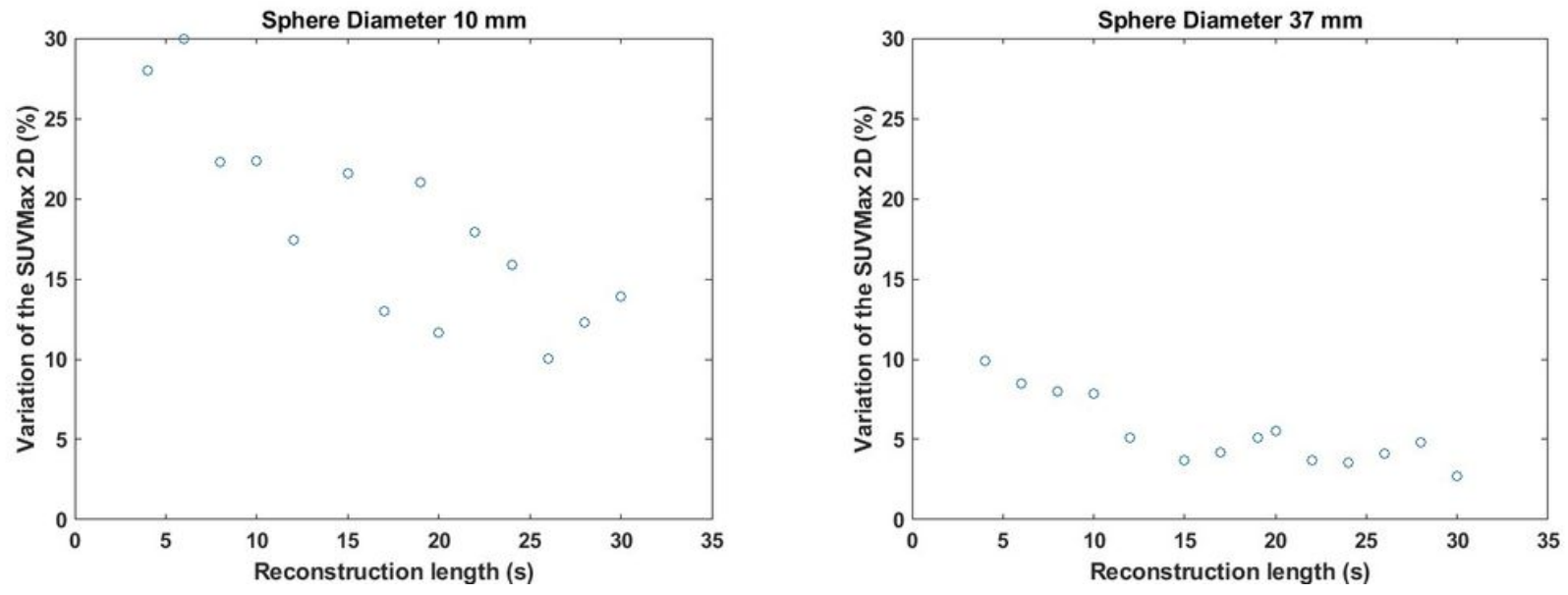

\section{Figure 2}

Coefficient of variation of the SUVMax 2D as a function of the reconstruction length. 

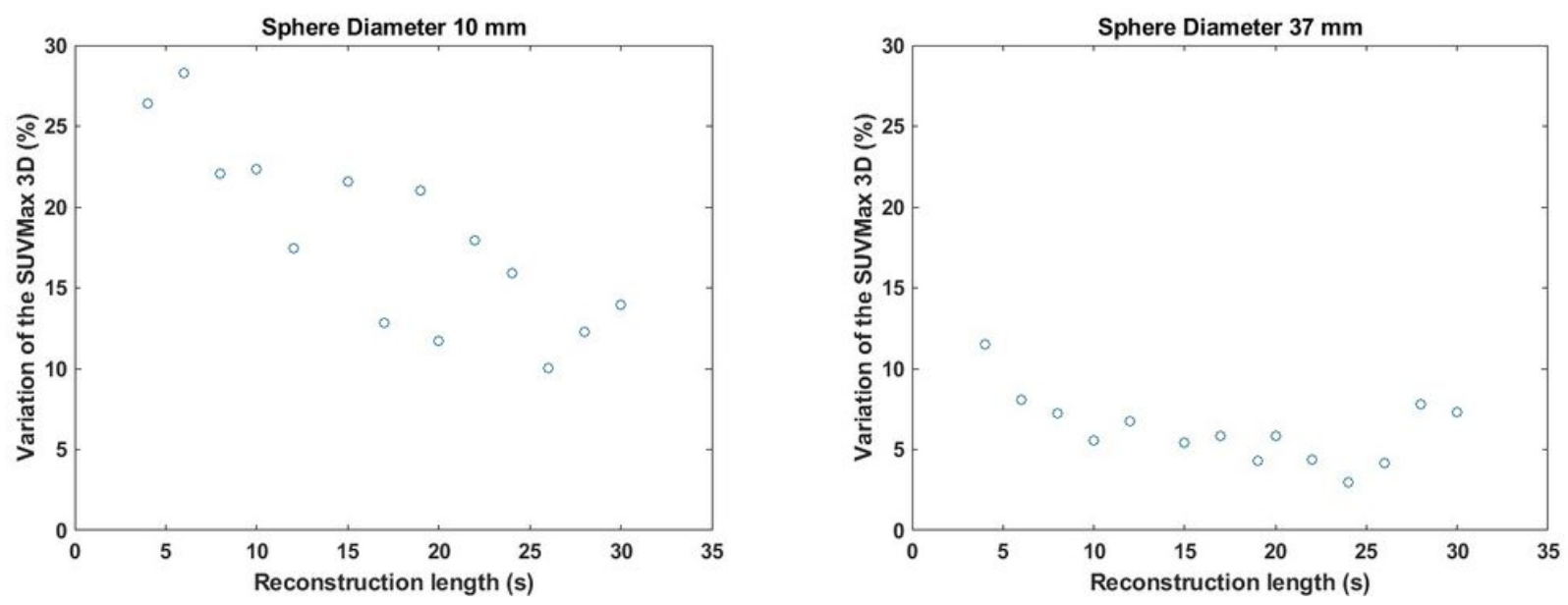

\section{Figure 3}

Coefficient of variation of the SUVMax 3D as a function of the reconstruction length.
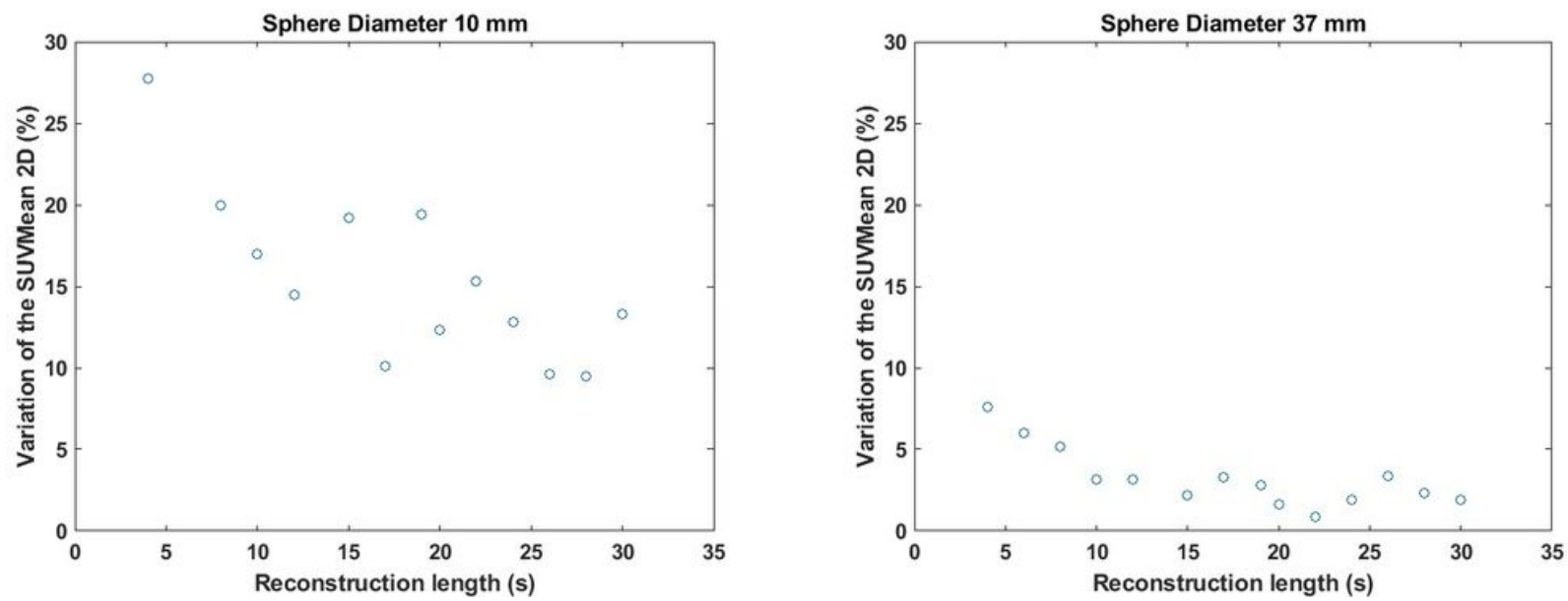

Figure 4

Coefficient of variation of the SUVMean 2D as a function of the reconstruction length.
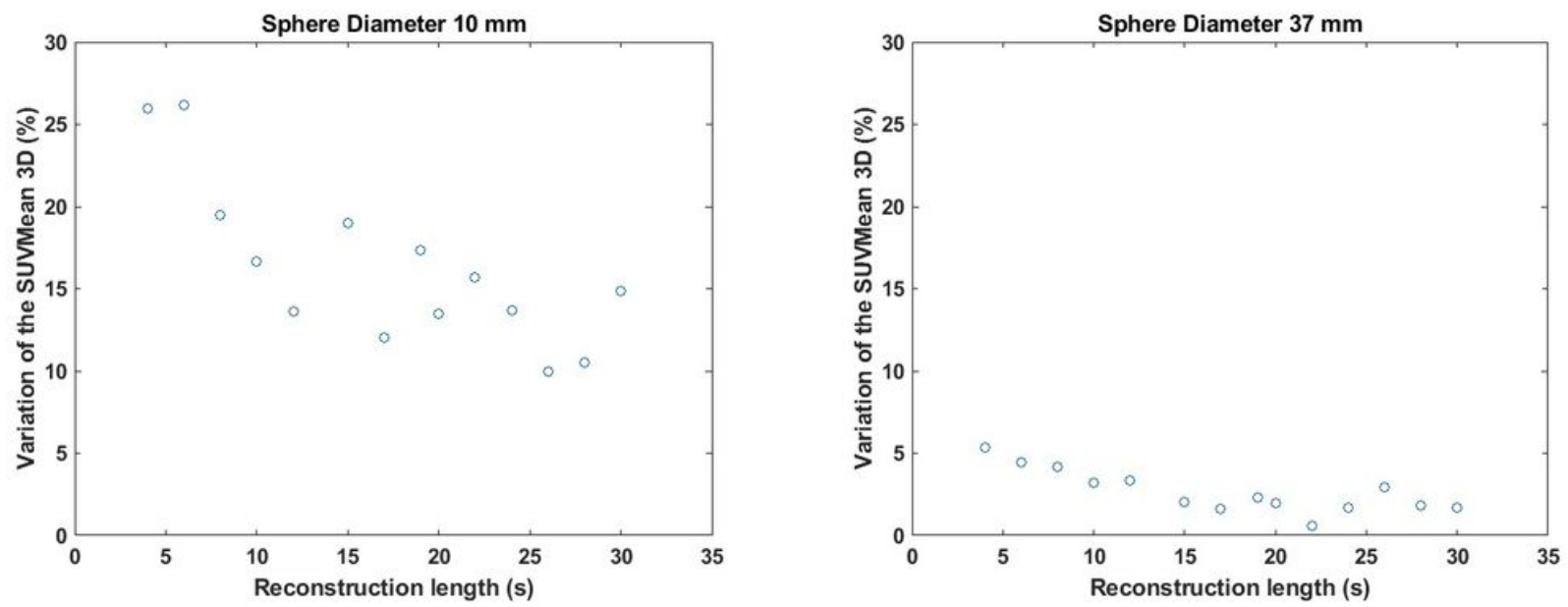

Figure 5

Coefficient of variation of the SUVMean 3D as a function of the reconstruction length. 

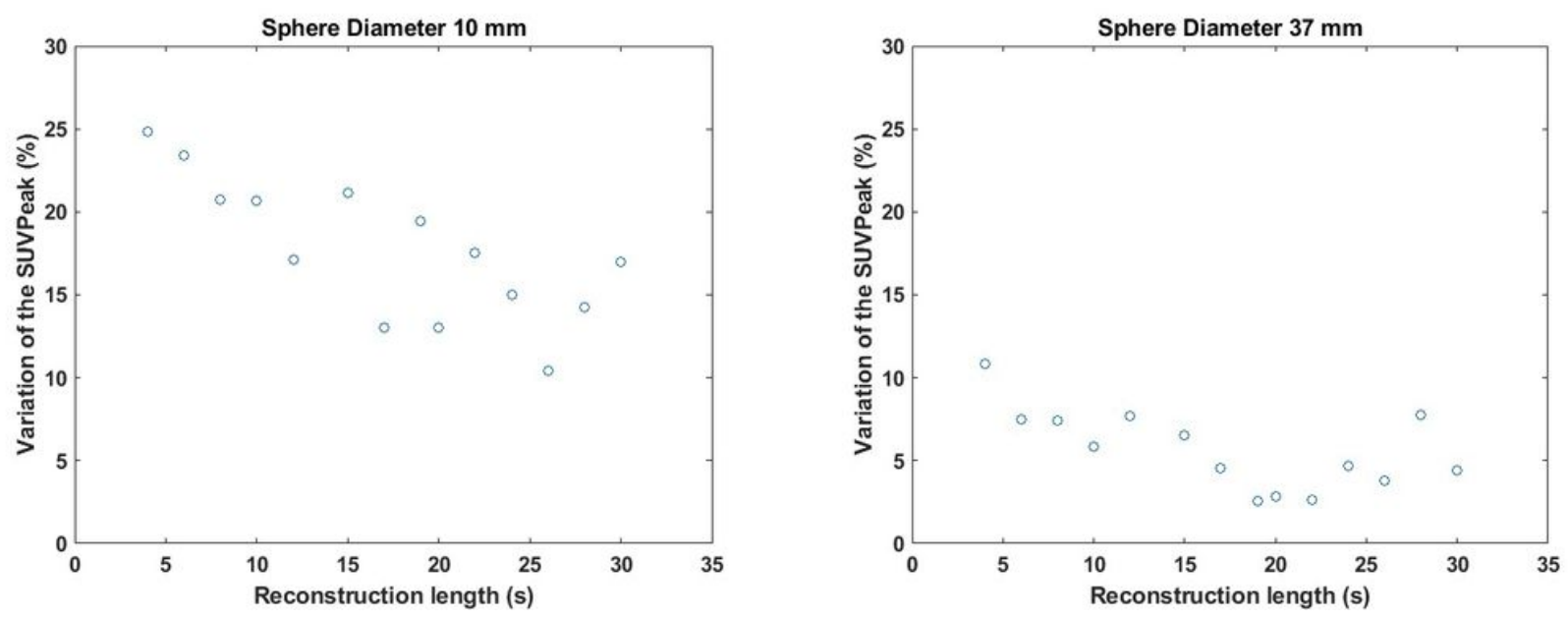

Figure 6

Coefficient of variation of the SUVPeak as a function of the reconstruction length.
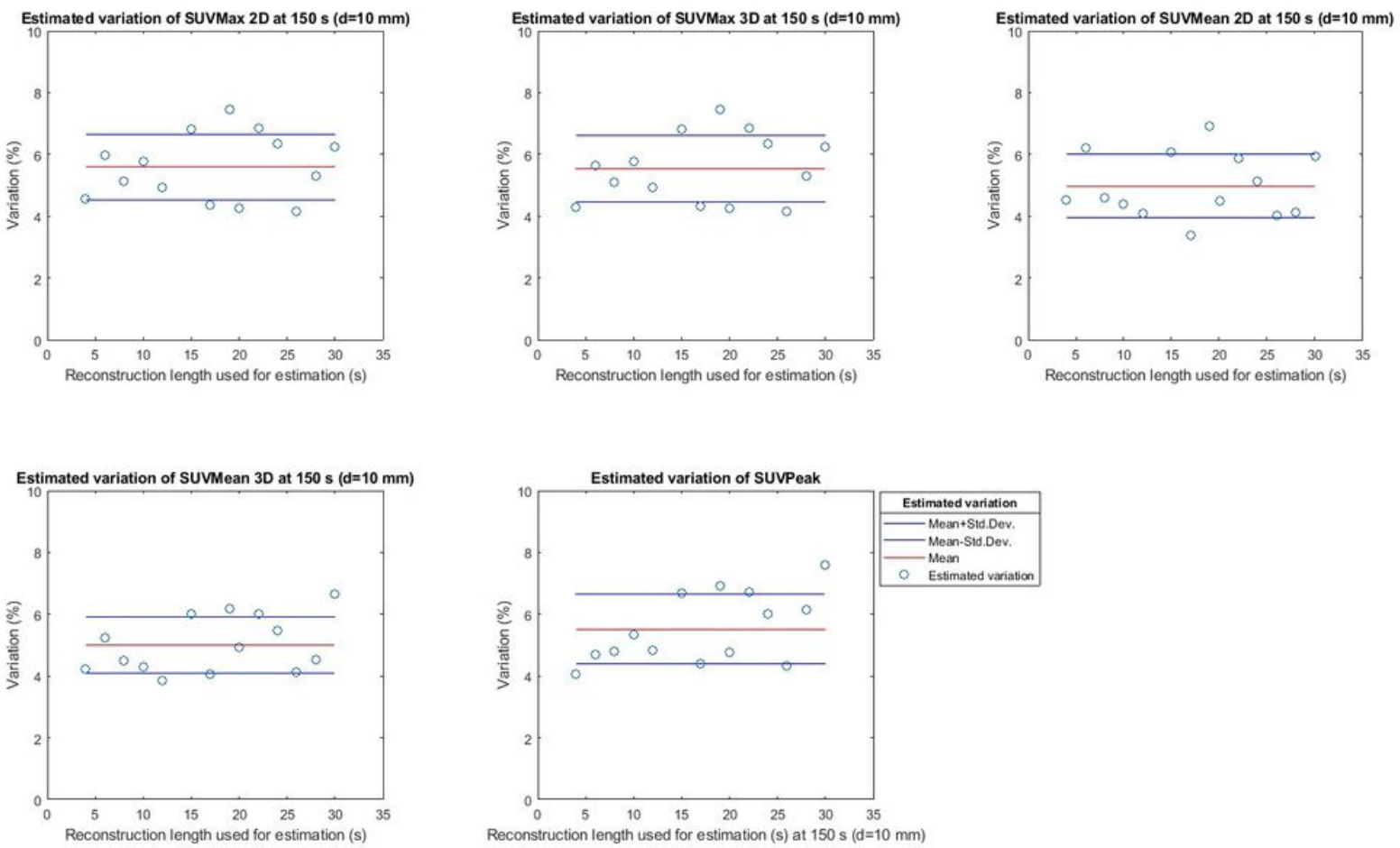

\section{Figure 7}

Estimated variation of the SUV parameters between repetitions of images with reconstruction length $150 \mathrm{~s}$ for a sphere of $10 \mathrm{~mm}$. 

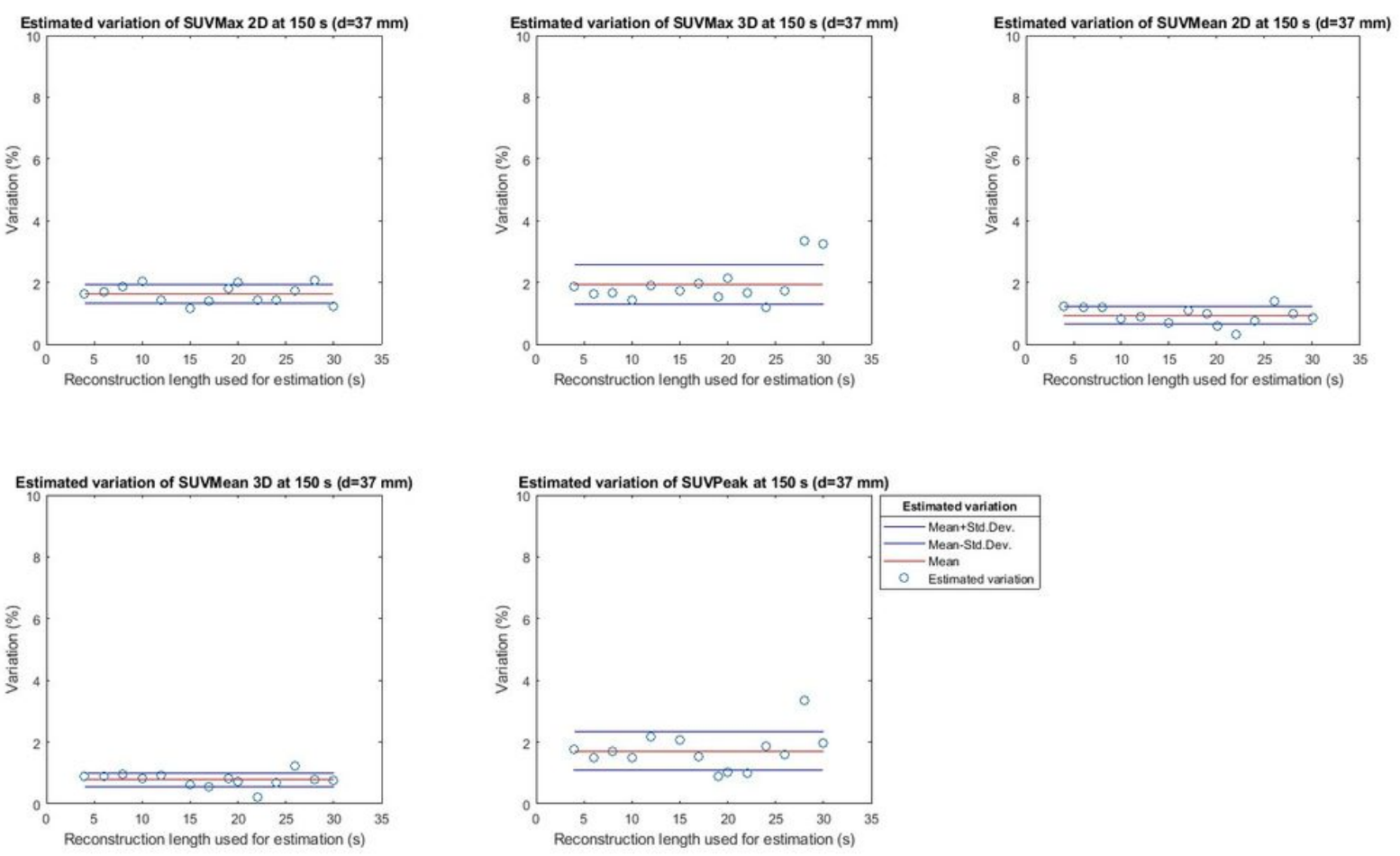

\section{Figure 8}

Estimated variation of the SUV parameters between repetitions of images with reconstruction length $150 \mathrm{~s}$ for a sphere of $37 \mathrm{~mm}$. 